\title{
Intussuscepção intestinal em gestante pós by- pass gástrico: relato de caso
}

Felipe Grion Trevisane ${ }^{1}$ (D), Tiago Ferrari ${ }^{1}$ (D), Eduardo Henrique Stefano ${ }^{1}$ (D), Guilherme Telles Hahn ${ }^{1}$ Nathalia Grion Trevisane ${ }^{2}$ (D), Daniele Fernanda Felipe ${ }^{3}$ (D), Ariana Ferrari $^{3}$

\begin{abstract}
RESUMO
Em todo o mundo, estima-se que aproximadamente 634.897 pessoas são submetidas a procedimentos bariátricos a cada ano, com gastrectomia vertical e by-pass gástrico, representando $53,6 \%$ e $30 \%$, respectivamente. Entre pacientes operados, aproximadamente metade são mulheres em idade reprodutiva. Em adultos, a intussuscepção é tipicamente devida a um local patológico no intestino, que pode ser maligno em mais da metade dos casos. A intussuscepção intestinal é rara em adultos, representando 1 a $5 \%$ das obstruções intestinais mecânicas. A intussuscepção em gestantes pós by-pass gástrico é uma patologia muito rara de origem desconhecida com alto poder de lesão e injúria de órgãos e sistemas. Suas manifestações clínicas são como abdômen agudo obstrutivo, porém, na paciente gestante dificulta o diagnóstico precoce. Este relato de caso apresenta uma paciente de 38 anos, gestante de 22 semanas, com quadro de dor abdominal de forte intensidade acompanhada de vômitos em grande quantidade há um dia, antecedente de by-pass gástrico há quatro anos. O diagnóstico do quadro de intussuscepção intestinal foi possível após o sétimo dia de internação com auxílio de exame de imagem - RNM, sendo indicado o tratamento cirúrgico para correção do caso. Evoluiu com choque séptico com necessidade de internação prolongada em leito de CTI, recebendo alta após 27 dias de internação.
\end{abstract}

Palavras-chave: Videolaparoscopia, Gestação, By-pass gástrico, Intussuscepção.

\section{INTRODUÇÃO}

A crescente demanda e popularidade das cirurgias bariátricas não se deve apenas à obesidade como uma epidemia, mas também para combater as comorbidades relacionadas à doença, como diabetes e hipertensão ${ }^{1}$. No entanto, a cirurgia bariátrica não está livre de complicações. A cirurgia bariátrica nas últimas décadas surgiu como tratamento mais eficaz para a obesidade mórbida, sendo capaz de proporcionar perda de peso, resolução ou melhoria das complicações da obesidade, em uma porcentagem consistente de pacientes, melhorando a expectativa e a qualidade de vida desses pacientes. Além disso, avanços técnicos e o aumento da experiência dos cirurgiões permitiram o uso generalizado de técnica minimamente invasiva para procedimentos ba- riátricos, diminuição do tempo pós-operatório e morbimortalidade ${ }^{2}$.

Em todo o mundo estima-se que aproximadamente 634.897 pessoas são submetidas a procedimentos bariátricos a cada ano. Dentre as diversas técnicas de cirurgia de obesidade, a gastrectomia vertical corresponde a $53,6 \%$ e o by-pass gástrico $30 \%{ }^{2}$. Acerca da distribuição, considera-se que o número de operados do sexo masculino seja o mesmo do feminino. Todavia, ao pensarmos nas mulheres, quase sua totalidade está em idade reprodutiva ${ }^{1}$.

Os benefícios da cirurgia bariátrica em pacientes do sexo feminino em idade reprodutiva incluem a melhoria da fertilidade e redução de risco de complicações obstétricas graves, como hipertensão induzida pela gravidez, pré-eclâmpsia, diabetes gestacional, macrossomia e cesariana ${ }^{1}$.

\footnotetext{
${ }^{1}$ Hospital Santa Rita de Maringá, Maringá, (PR), Brasil.

2 Universidade Estadual Paulista (UNESP), Botucatu, (SP), Brasil.

${ }^{3}$ Universidade Cesumar (Unicesumar). Programa de Mestrado em Tecnologias Limpas, Maringá, (PR), Brasil.
} 
Gestantes com histórico de cirurgia bariátrica devem ser consideradas um grupo de alto risco, exigindo atenção especial durante o pré-natal. De fato, esse subconjunto de pacientes é exposto por possíveis complicações médicas e cirúrgicas. As complicações médicas incluem deficiências de micronutrientes, o aumento do risco de prematuridade e intussuscepção intestinal durante a gestação, como complicação cirúrgica ${ }^{1}$.

Em adultos, a intussuscepção é tipicamente devida a um local patológico no intestino, como, por exemplo, um tumor, que age como ponto fixo em um ambiente previamente livre. Dessa forma, através do peristaltismo fisiológico do delgado, a alça proximal acaba por invaginar neste local ${ }^{3}$.

No entanto, em alguns casos, a intussuscepção pode ser classificada por etiologia como lesão benigna por pólipos, divertículo de Meckel ou de caráter idiopático ${ }^{3}$. Sobre sua localização, é classificada em alças intestinais entero-entéricas, quando limitada ao intestino delgado; íleo-cólica com prolapso do íleo terminal no cólon ascendente; e colo-cólica, que é limitada ao intestino grosso ${ }^{3}$.

A intussuscepção intestinal é rara em adultos, representando 1 a $5 \%$ das obstruções intestinais mecânicas ${ }^{3}$. A maior incidência de intussuscepção é vista em pacientes com síndrome da imunodeficiência adquirida (SIDA). Isso se deve à alta incidência de doenças infecciosas e neoplásicas do intestino em pacientes com SIDA, como hiperplasia linfoide, sarcoma de Kaposi e linfoma não-Hodgkin. E em crianças com divertículo de Meckel $^{1}$.

Nos adultos, a dor abdominal intermitente é a apresentação mais comum; no entanto, os pacientes também podem apresentar sintomas consistentes com obstrução intestinal parcial, com náusea, vômitos, melena, perda de peso, febre e constipação. O quadro clínico do paciente com intussuscepção intestinal é o de um abdômen agudo obstrutivo ${ }^{3}$.

$O$ exame de raio $x$ de abdômen simples pode mostrar características típicas de obstrução do intestino delgado, com dilatação de alças intestinais, ausência de ar na localização do reto, sinal de "empilhamento de moedas" e nível hidroaéreo 4 .

O diagnóstico, todavia, geralmente é feito na tomografia de abdominal. A alça distendida do intestino se apresenta espessada porque repre- senta duas camadas de intestino. Um "sinal alvo" pode ser visto na incidência sagital da tomografia abdominal, enquanto na vista axial ou coronal, a intussuscepção aparecerá como uma massa em forma de salsicha ${ }^{5}$.

$\mathrm{Na}$ paciente gestante prefere-se o uso de ressonância magnética sem contraste endovenoso para evitar os riscos da radiação para o feto, apresentando sensibilidade quase igual a tomografia de abdômen ${ }^{6}$. O tratamento definitivo consiste em abordagem cirúrgica por via laparotômica ou laparoscópica. Na abordagem inicial do paciente pode ser necessário o uso de sonda nasogástrica aberta para diminuir a pressão intragástrica e intraluminal das alças intestinais com medicações para analgesia e antieméticos, até a abordagem cirúrgica adequada do paciente ${ }^{1}$. A técnica cirúrgica consiste no desenluvamento da alça intestinal sobre seu próprio lúmen de maneira delicada, observando possíveis pontos de sofrimento isquêmico da parede intestinal compatíveis com necrose. Quando há sofrimento isquêmico de parede, deve-se proceder uma enterectomia no ponto obstruído com necessária anastomose nas alças intestinais residuais, mecânica ou manual ${ }^{2}$.

Alguns pacientes com casos de intussuscepção em alças colônicas podem se beneficiar de tratamento com enema opaco por via transretal, não sendo necessário assim uma abordagem cirúrgica. Porém, o índice de sucesso com este tratamento não tem sido favorável, sendo uma conduta pouco vista na prática ${ }^{3}$.

Embora a redução da intussuscepção seja segura e eficaz, existe um risco significativo de recorrência. Ressecção, anastomose de ponta a ponta, do ponto de obstrução parece ser eficaz como meio de tratamento, evoluindo para um bom prognóstico na maioria dos casos ${ }^{1}$.

\section{RELATO DE CASO}

Paciente, sexo feminino, 38 anos, gestante com 22 semanas, previamente hígida, admitida no pronto atendimento para gestantes do Hospital Santa Rita de Maringá no município de Maringá/PR, com quadro de dor abdominal súbita em região de abdômen superior iniciada há um dia, irradiada para dorso associado a náuseas. Apre- 
sentou melhora do quadro de dor ao uso de morfina endovenosa.

Durante a investigação diagnóstica foi relatado histórico de by-pass gástrico e colecistectomia há nove anos, cesárea e apendicectomia prévia. Sem comorbidades associadas. Exames laboratoriais e ultrassonográfico de abdômen superior, durante admissão, foram normais. Dessa forma, a equipe optou pelo internamento da paciente, para melhor investigação da dor abdominal. Durante a internação foi solicitada endoscopia digestiva alta após evoluir com hematêmese, que evidenciou sangramento em trato gastrointestinal superior, todavia, sem lesão visualizada, com dificuldade de progressão do endoscópio devido cirurgia gástrica prévia.
Na rotina de exames laboratoriais apresentou aumento de amilase sérica maior que três vezes o valor sérico no segundo dia de internação, em que foi aberta a hipótese diagnóstica de pancreatite aguda grave, necessitando transferência para leito da unidade de terapia intensiva.

Sem melhora do quadro de dor abdominal no quinto dia de internação e sem melhora dos exames laboratoriais, incluindo amilase, foi indicada a realização de ressonância magnética com visualização de ponto obstrutivo ao exame de imagem (Imagem 1). Apesar de sinal sugestivo de intussuscepção, a equipe optou por manter tratamento conservador, pensando na viabilidade fetal.

Imagem 1: "Sinal em alvo", sugestivo de intussuscepção intestinal.

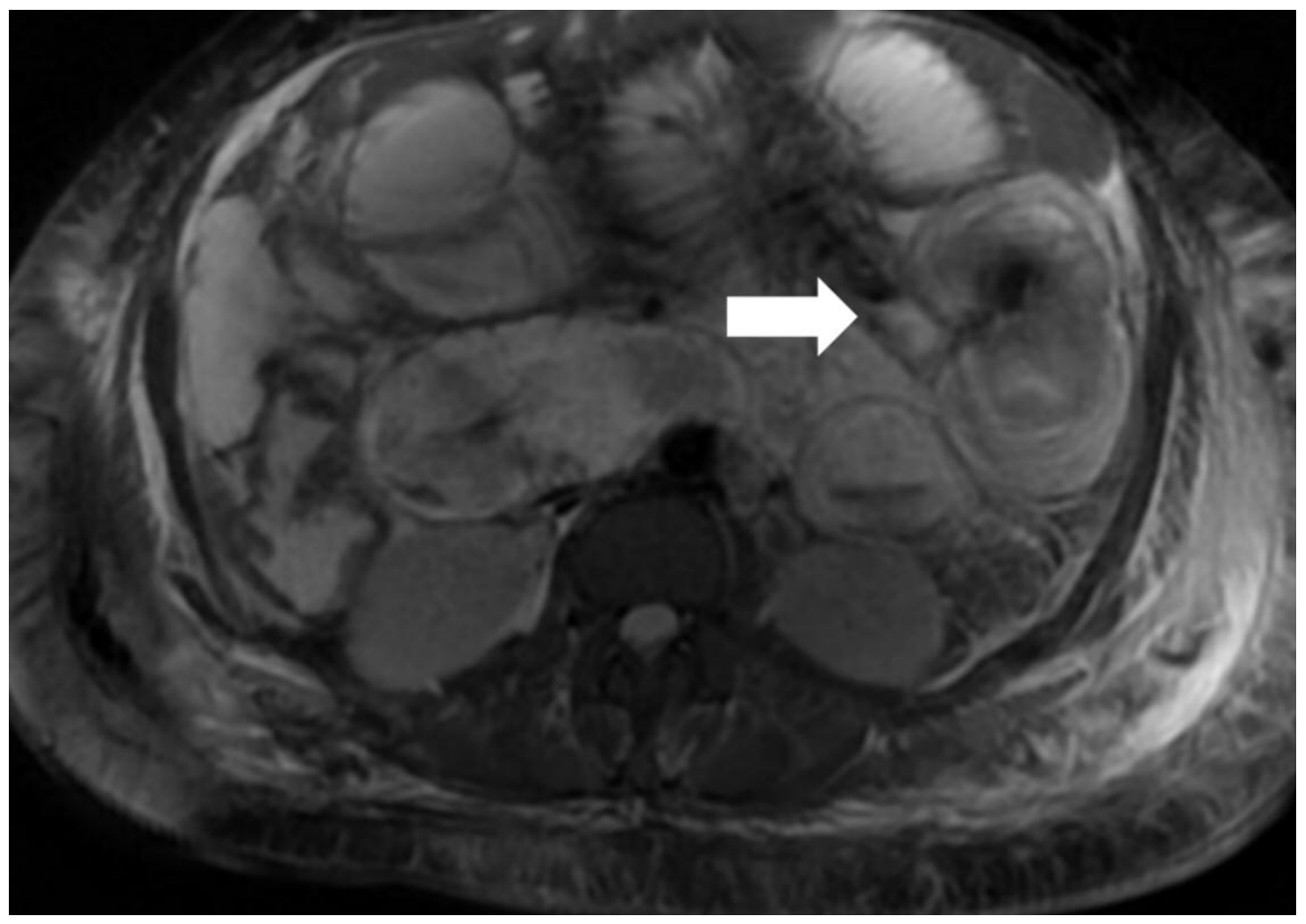

Todavia, no sétimo dia de internação evoluiu com instabilidade hemodinâmica, sendo necessário iniciar drogas vasoativas e intubação orotraqueal para estabilização do quadro.

Devido manutenção do quadro clínico grave e drogas vasoativas em alta doses, foi optado pela realização de uma tomografia de abdômen total que evidenciou obstrução intestinal mecânica, sendo indicado laparotomia de emergência.
Durante a cirurgia, foi confirmada a intuscepção de alça comum do $Y$ de Roux sobre a alça biliar (Imagem 2), com perfuração do estômago excluso. Foi necessária a resseção cirúrgica da intussuscepção com a reconstrução do trânsito intestinal por entero-entero anastomose e gastrostomia em estômago excluso para diminuição da pressão no trato gastrointestinal. 
Imagem 2: Peça anatômica pós ressecção cirúrgica.

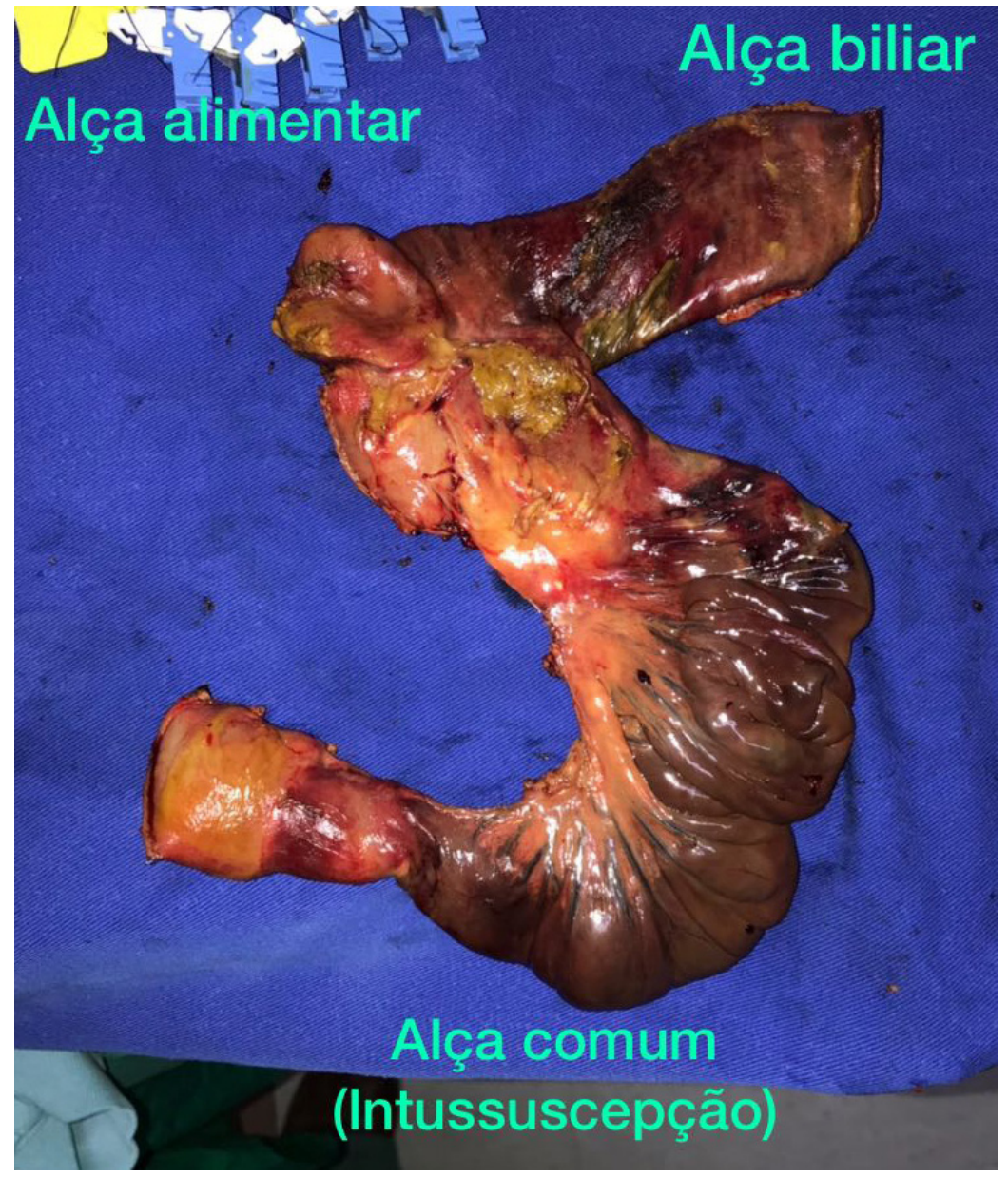

A paciente evoluiu com expulsão de feto em óbito no pós-operatório imediato, no leito da unidade de terapia intensiva, sendo necessário manter o uso de drogas vasoativas devido grande instabilidade hemodinâmica.

Durante a internação na unidade de terapia intensiva, se manteve instável por um longo período, sendo necessária diálise prisma para melhora da função renal e estabilidade hemodinâmica.

Foi realizada nova laparotomia durante a internação para lavagem de cavidade abdominal e drenagem de abcesso abdominal em região pélvica, onde as novas anastomoses se mantinham intactas sem alterações. O suporte pós-operatório foi mantido em unidade de terapia intensiva.

Após 21 dias de internação em leito da unidade de terapia intensiva recebeu alta para o quarto e alta domiciliar no $27^{\circ}$ dia de internação, em bom estado geral, apenas com receita médica de medicações sintomáticas. Mantém acompanhamento via ambulatorial desde o sétimo dia de alta hospitalar, sem novas queixas desde então.

\section{DISCUSSÃO}

A complicação intestinal de abdômen agudo obstrutivo que ocorre após a cirurgia de by-pass geralmente é decorrente de aderências de alças intestinais, hérnias internas ou intussuscepção ${ }^{7}$, como mostrado no caso citado.

Apenas poucos casos de abdômen agudo obstrutivo pós by-pass gástrico em pacientes ges- 
tantes têm sido relatados na literatura. Sugere que o aumento da pressão intra-abdominal associado a redução abrupta de peso contribua para um maior risco de complicações, principalmente no segundo e terceiro trimestres de gestação ${ }^{8}$. Não se pode dizer com clareza que a gravidez pode aumentar o risco de intussuscepção intestinal, mas uma redução de peso acentuada aumenta a chances de torção e intussuscepção intestinal ${ }^{2}$. Estudos indicam que a maior incidência ocorre após quatro anos do procedimento cirúrgico ${ }^{1}$. No caso relatado, a paciente apresentava um período de nove anos da cirurgia de by-pass gástrico, corroborando a hipótese.

Com idade gestacional de 22 semanas, os artigos mostram uma idade média que envolve 27,42 semanas, sendo o caso apresentado precoce, mas já dentro de uma faixa gestacional em que o útero aumenta a pressão intra-abdominal ${ }^{2}$.

As náuseas e vômitos da gestação muitas vezes podem atrapalhar o diagnóstico da intussuscepção intestinal, e, aliado a dores abdominal inespecíficas da gravidez, tornam o limiar para suspeita de qualquer complicação da cirurgia de by-pass baixo².

Já no diagnóstico da intussuscepção, o exame de escolha é a tomografia de abdômen, porém, na paciente gestante, traz riscos para o feto, sendo assim, o primeiro exame a ser pedido a $\mathrm{RNM}^{9}$, o que foi realizado no caso da paciente. Todavia, pensando em preservar a integridade do feto, culminou em um atraso no tratamento cirúrgico.

Esse atraso no diagnóstico aumenta exponencialmente o risco de morte fetal. Estudos mostram que o atraso em 48 horas aumenta em $10 \%$ a taxa de morte fetal, ao passo que mais de 48 horas de atraso na intervenção atingiria $50 \%$ de probabilidade de óbito ${ }^{10}$. No caso apresentado, além do atraso na exploração cirúrgica, aliou-se o uso de drogas vasoativas em altas doses, sedativos, devido a intubação orotraqueal, com o desfecho sendo o aborto espontâneo apresentado pela paciente.

\section{CONCLUSÃO}

Concluímos que a intussuscepção intestinal em gestante pós by-pass gástrico é uma patologia muito rara e, embora possua diversos elementos que possam contribuir para sua gênese, permanece sendo considerada de origem desconhecida, com alto poder de lesão e injúria de órgãos e sistemas. Em sua maioria, apresenta difícil diagnóstico em um primeiro momento, pois a gestação por si só apresenta sinais e sintomas semelhantes aos de abdome agudo obstrutivo, como vômitos e dor abdominal difusa, tornando o diagnóstico diferencial entre patologia de conduta conservadora ou cirúrgica, desafiador.

As baixas incidências da doença, associada à dificuldade no diagnóstico geraram uma deficiência de análises literárias de séries de casos, fazendo com que a produção científica acerca da enfermidade seja insuficiente. Aliado a esses fatores, o fato de o médico sempre pensar em preservar o feto faz com que muitas condutas cirúrgicas sejam adiadas, trazendo injúria ainda maior tanto para mãe quanto para o feto. Desse modo, nota-se que a descrição científica de mais um caso de intussuscepção intestinal em gestante pós by-gástrico pode contribuir para a riqueza de dados científicos acerca desta patologia e, consequentemente, com o embasamento teórico para a tomada de decisão clínica dos estudantes e profissionais médicos, visando o melhor prognóstico para o paciente.

O estudo confirma a importância sobre a orientação e ênfase sobre os riscos e complicações inerentes após o by-pass gástrico a longo prazo, já que as complicações na paciente gestante elevam o risco de óbito fetal e materno.

\section{REFERÊNCIAS}

1. Boccalatte LA, Achaval Rodríguez J, Beskow A, Cavadas $D$, Fernando W. Intussusception as a complication of bariatric surgery in pregnant patients: report of one case and revision of the literature. J Surg Case Rep. 2017;2017(10): rjx189.

2. Boudourakis LD, Divino C, Nguyen S. Retrograde intussusception seven years after a laparoscopic Roux-en-Y gastric bypass. J Minim Access Surg. 2013;9(2):82-83.

3. Bordeianou $L$, Yeh DD, Etiologies, clinical manifestations, and diagnosis of mechanical small bowel obstruction in adults. Uptodate.com 2020. UpToDate. Waltham, MA: UpToDate Inc. https://www.uptodate.com (Accessed on July 07, 2020.)

4. Marinis $A$, Yiallourou $A$, Samanides $L$, et al. Intussusception of the bowel in adults: a review. World J Gastroenterol. 2009;15(4):407-411. 
5. Honjo $H$, Mike $M$, Kusanagi $H$, Kano $N$. Adult intussusception: a retrospective review. World J Surg. 2015;39(1):134-138.

6. Gayer G, Zissin R, Apter S, Papa M, Hertz M. Pictorial review: adult intussusception--a CT diagnosis. $\mathrm{Br}$ J Radiol. 2002;75(890):185-190.

7. Moore KA, Ouyang DW, Whang EE. Maternal and fetal deaths after gastric bypass surgery for morbid obesity. $\mathrm{N}$ Engl J Med 2014; 351:721-2.

8. Tuyeras G, Pappalardo E, Msika S. Acute small bowel obstruction following laparoscopic Roux-en-Y gastric bypass during pregnancy: two different Presentations. J Surg Caser Rep 2012; 2012:1

9. Singla S, Guenthart BA, May L Et al. Intussusception after laparoscopic gastric bypass surgery: an underrecognized complication. Minim Invasive Surg 2012; 2012:464853.

10. Bellón M. Coexistencia de dos complicaciones frecuentes tras Bypass Gástrico. 5.3.11 (886-889). www.bmilatina. com, dic. 2015.

Autor Correspondente:

Ariana Ferrari

ariana.ferrari@unicesumar.edu.br

Editor:

Prof. Dr. Felipe Villela Gomes

Recebido em: 11/07/2020

Aprovado em: 07/10/2020 